DOI: $10.15393 /$ j3.art.2021.10030

UDC 517.968, 517.98, 51-75

M. BOUlanouar

\title{
MATHEMATICAL ANALYSIS OF A MODEL OF AGE-CYCLE LENGTH STRUCTURED CELL POPULATION WITH QUIESCENCE
}

\begin{abstract}
In this work, we model the dynamics of an Age-Cycle Length structured cell population. At each time, the cell population is divided into two interacting compartments: Proliferating cells and Quiescent cells. Each cell is then: Proliferating (Active) or Quiescent (Resting). We prove that this new Proliferation-Quiescence model is well posed.
\end{abstract}

Key words: Partial Differential Equations, Semigroup of Linear Operators, Structured Cell Population

2010 Mathematical Subject Classification: 45K05; 47D06; $92 C 17$

1. Introduction. In this work we model the dynamics of a Cell Cycle Length-Age structured cell population. At each time, the cell population is divided into two interacting compartments: Proliferating cells (P) and Quiescent cells (Q). Then each cell is either Proliferating (Active) or Quiescent (Resting).

Quiescence (also called $G_{0}$ ) is the most common cell state on Earth. It is the counterpart to proliferation: a reversible and nondividing state. For instance, cells in uninjured skin, adult neuronal cells, cells of the adult mammalian heart, somatic cells, .... All these cells, and so many others, are quiescent.

So, let us consider a cell population in which each cell is distinguished by two physiological parameters. The first one is the cell cycle length $l \in\left(l_{1}, l_{2}\right)\left(0 \leqslant l_{1}<l_{2} \leqslant \infty\right)$. It describes the time between cell's birth and cell's mitosis (or division). The second one is the age $a$. It is zero $(a=0)$ at birth and equals the cell cycle length $l(a=l)$ at mitosis. Between birth and mitosis, we have $0 \leqslant a \leqslant l$. Before writing the mathematical model, let us put the following biological assumptions:

(c) Petrozavodsk State University, 2021 
Assumption 1. Assume that in the Proliferation Phase (P) cells are born, grow, and divide. They carry out their life processes and then they die (by mitosis or other causes).

Assumption 2. Assume that after the birth cells go into the Quiescence Phase (Q). In this phase, cells remain metabolically active but do not proliferate and do not undergo any kind of division.

Assumption 3. Each cell is fully characterized by its status: Proliferating (Active) or Quiescent (Resting). Cells can transit back and forth from one state to the other. Cells transit between the two phases is described by the following scheme

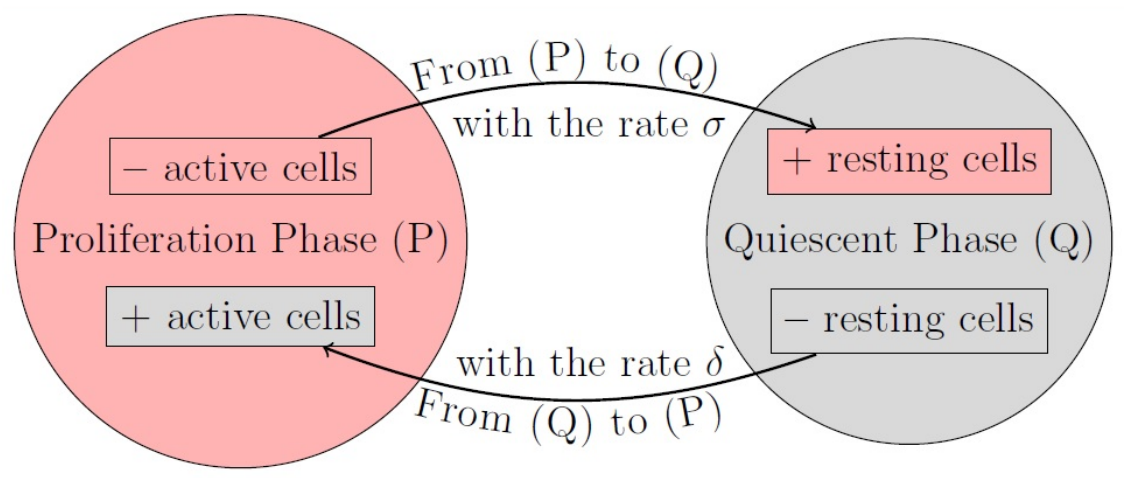

Figure 1: Diagram of the cell transit between $(\mathrm{P})$ and $(\mathrm{Q})$.

where $\sigma$ and $\delta$ denote, respectively, the transition rates from the Proliferation phase $(\mathrm{P})$ to the Quiescence phase $(\mathrm{Q})$, and vice versa. Let $(p, q)=(p(t, a, l), q(t, a, l))$ denote, at time $t$, the density of proliferating and quiescent cells, with respect to the age $a$ and cell cycle length $l$. According to the Figure above, we write

$$
\begin{gathered}
\frac{\partial p}{\partial t}+\frac{\partial p}{\partial a}+\mu p-\int_{l_{1}}^{l_{2}} \eta\left(a, l, l^{\prime}\right) p\left(t, a, l^{\prime}\right) \mathrm{d} l^{\prime}=-\sigma p+\delta q, \\
\frac{\partial q}{\partial t}+\frac{\partial q}{\partial a}=+\sigma p-\delta q,
\end{gathered}
$$

where $\mu=\mu(a, l)$ denotes the mortality rate in the Proliferation phase. The kernel $\eta\left(a, l, l^{\prime}\right)$ denotes the transition rate in which proliferating cells change their cell cycle length from $l^{\prime}$ to $l$. 
According to Assumption 1, proliferating cells divide. At each mitosis, the cell population is divided in two distinct subpopulations in most observed cases. In the first subpopulation, there is a total inheritance of the cell cycle length $l$ between a mother cell and its daughters, while in the second one there is a correlation $\mathrm{k}=\mathrm{k}\left(l, l^{\prime}\right)$ between the cell cycle length, $l^{\prime}$, of the mother cell and that of a daughter cell, $l$. Hence,

$$
p(t, 0, l)=\alpha p(t, l, l)+\beta \int_{l_{1}}^{l_{2}} \mathrm{k}\left(l, l^{\prime}\right) p\left(t, l^{\prime}, l^{\prime}\right) \mathrm{d} l^{\prime}, l \in\left(l_{1}, l_{2}\right),
$$

where $\alpha \geqslant 0$ and $\beta \geqslant 0$ denote the average number of daughter cells viable per mitosis into the corresponding subpopulation.

However, Assumption 2 means that the quiescent cells do not divide. Therefore,

$$
q(t, 0, l)=0, \quad l \in\left(l_{1}, l_{2}\right)
$$

The simplified proliferation model (1) (with $\sigma=\delta=\eta=0$ ) and (3) (with $\alpha=0$ ) has been studied (see [4], [6] and references therein) when $0<$ $<l_{1}<l_{2}<\infty$. We have recently improved it by introducing the transition rate $\eta\left(a, l, l^{\prime}\right)$ like in [5]. We have proved, then, that the new proliferation model (1) (with $\sigma=\delta=0$ ) and (3) is governed by a $C_{0}$-semigroup when $0<l_{1}<l_{2}<\infty$ (see [2], [3]).

The purpose of this work is to analyze the new full ProliferationQuiescence Model (1)-(4) when $0 \leqslant l_{1}<l_{2} \leqslant \infty$; that is,

$$
\begin{array}{lr}
\frac{\partial p}{\partial t}=-\frac{\partial p}{\partial a}-\sigma p+\delta q-\mu p+\int_{l_{1}}^{l_{2}} \eta\left(a, l, l^{\prime}\right) p\left(t, a, l^{\prime}\right) \mathrm{d} l^{\prime}, & (\mathrm{PQ})_{1} \\
\frac{\partial q}{\partial t}=-\frac{\partial q}{\partial a}+\sigma p-\delta q, & (\mathrm{PQ})_{2} \\
p(t, 0, l)=\alpha p(t, l, l)+\beta \int_{l_{1}}^{l_{2}} \mathrm{k}\left(l, l^{\prime}\right) p\left(t, l^{\prime}, l^{\prime}\right) \mathrm{d} l^{\prime}, & (\mathrm{PQ})_{3} \\
q(t, 0, l)=0, & (\mathrm{PQ})_{4}
\end{array}
$$

describing a structured cell population with two interacting compartments: Proliferating cells (P) and Quiescent cells (Q). To our knowledge, this model is new and has never been proposed nor studied. We organize this work as follows: 


\section{Trace Result;}

3. Unperturbed Quiescence Model;

4. Unperturbed Proliferation Model;

5. Unperturbed Proliferation-Quiescence Model;

6. Full Proliferation-Quiescence Model.

In Section 2, we prove a Trace Result. This one allows us to make a sense to all unbounded linear operators considered in this work. Section 3 deals with the unperturbed Quiescence Model $(\mathrm{PQ})_{2}($ with $\sigma=\delta=0)$ and $(\mathrm{PQ})_{4}$. We prove then that this Model is governed by a $C_{0}-$ semigroup of contractions. This contractiveness is due to Assumption 2.

In Section 4, we consider the unperturbed Proliferation Model (PQ) (with $\mu=\sigma=\delta=\eta=0$ ) and (PQ) $)_{3}$. The case $l_{1}=0$ means that there are cells that are born, simultaneously, as mothers and daughters. Therefore, we study the two cases $l_{1}>0$ and $l_{1}=0$ separately whenever this is necessary. In each case, we prove that the considered unperturbed Proliferation Model is governed by a $C_{0}$-semigroup provided a suitable assumption on the kernel of correlation $\mathrm{k}$ holds.

Section 5 deals with the unperturbed-Proliferation Quiescence Model $(\mathrm{PQ})_{1}($ with $\mu=\sigma=\delta=\eta=0),(\mathrm{PQ})_{2}($ with $\sigma=\delta=0),(\mathrm{PQ})_{3}$ and $(\mathrm{PQ})_{4}$. Using the results of the previous sections, we prove that the considered unperturbed Proliferation-Quiescence Model is governed by a $C_{0}$-semigroup.

Finally, in Section 6 we consider the full Model $(\mathrm{PQ})_{1}-(\mathrm{PQ})_{4}$. According to relevant assumptions on the rates, $\mu, \sigma, \delta$, and on the kernel $\eta$, the full Proliferation-Quiescence Model $(\mathrm{PQ})_{1}-(\mathrm{PQ})_{4}$ appears then as a linear perturbation of the unperturbed Proliferation-Quiescence Model already studied in Section 5; then the desired well-posedness of the full Proliferation-Quiescence Model $(\mathrm{PQ})_{1}-(\mathrm{PQ})_{4}$ follows. We end this work by some remarks.

2. Trace Result.The aim of this section is to prove a useful trace result allowing us to define all unbounded linear operators throughout this work. So, let $l_{1}$ and $l_{2}$ be such that $0 \leqslant l_{1}<l_{2} \leqslant \infty$ and let $\Omega:=\left\{(a, l): 0<a<l\right.$ and $\left.l_{1}<l<l_{2}\right\}$. Let $\mathrm{L}_{1}$ and $\mathrm{Y}_{1}$ be the following Banach spaces:

$$
\mathrm{L}_{1}:=L^{1}(\Omega) \quad \text { whose norm is } \quad\|\varphi\|_{1}:=\int_{\Omega}|\varphi(a, l)| \mathrm{d} a \mathrm{~d} l,
$$




$$
\mathrm{Y}_{1}:=L^{1}\left(l_{1}, l_{2}\right) \quad \text { whose norm is } \quad\|\psi\|_{\mathrm{Y}_{1}}:=\int_{l_{1}}^{l_{2}}|\psi(l)| \mathrm{d} l .
$$

Let also $\mathrm{W}_{1}$ be the following Banach space:

$$
\begin{aligned}
& \mathrm{W}_{1}:=\left\{\varphi \in \mathrm{L}_{1}: \frac{1}{l} \varphi \in \mathrm{L}_{1} \text { and } \frac{\partial \varphi}{\partial a} \in \mathrm{L}_{1}\right\} \\
& \text { normed by }\|\varphi\|_{\mathrm{W}_{1}}:=\|\varphi\|_{1}+\left\|\frac{\partial \varphi}{\partial a}\right\|_{1}+\left\|\frac{1}{l} \varphi\right\|_{1} .
\end{aligned}
$$

Lemma 1. Let $\gamma_{0}$ and $\gamma_{1}$ be such that

$$
\gamma_{0} \varphi(l):=\varphi(0, l) \quad \text { and } \quad \gamma_{1} \varphi(l):=\varphi(l, l), \quad l \in\left(l_{1}, l_{2}\right) .
$$

Then $\gamma_{0}$ and $\gamma_{1}$ are continuous mappings from $\mathrm{W}_{1}$ into $\mathrm{Y}_{1}$.

Proof. Let $\varphi \in \mathrm{W}_{1}$. For almost all $(a, l) \in \Omega$, we have

$$
\left|\gamma_{0} \varphi(l)\right|=\left|\varphi(a, l)-\int_{0}^{a} \frac{\partial \varphi}{\partial a}(s, l) \mathrm{d} s\right| \leqslant|\varphi(a, l)|+\int_{0}^{l}\left|\frac{\partial \varphi}{\partial a}(s, l)\right| \mathrm{d} s .
$$

Integrating with respect to $a(0<a<l)$ leads to

$$
\left|\gamma_{0} \varphi(l)\right| \leqslant \frac{1}{l} \int_{0}^{l}|\varphi(a, l)| \mathrm{d} a+\int_{0}^{l}\left|\frac{\partial \varphi}{\partial a}(s, l)\right| \mathrm{d} s .
$$

Hence

$$
\left\|\gamma_{0} \varphi\right\|_{\mathrm{Y}_{1}} \leqslant\left\|\frac{1}{l} \varphi\right\|_{1}+\left\|\frac{\partial \varphi}{\partial a}\right\|_{1} \leqslant\|\varphi\|_{\mathrm{W}_{1}}
$$

which proves that $\gamma_{0}$ is continuous from $\mathrm{W}_{1}$ into $\mathrm{Y}_{1}$. Since

$$
\gamma_{1} \varphi(l)=\varphi(a, l)+\int_{a}^{l} \frac{\partial \varphi}{\partial a}(s, l) \mathrm{d} s,
$$

we prove in a similar way that $\gamma_{1}$ is continuous from $W_{1}$ into $Y_{1}$.

Remark. If $l_{1}>0$, then $\left\|\frac{1}{l} \varphi\right\|_{1} \leqslant \frac{1}{l_{1}}\left\|\frac{1}{l} \varphi\right\|_{1}$ for all $\varphi \in \mathrm{L}_{1}$. In this case, the Banach space $\mathrm{W}_{1}$ becomes

$$
\mathrm{W}_{1}=\left\{\varphi \in \mathrm{L}_{1}: \frac{\partial \varphi}{\partial a} \in \mathrm{L}_{1}\right\}, \quad\|\varphi\|_{\mathrm{W}_{1}}:=\|\varphi\|_{1}+\left\|\frac{\partial \varphi}{\partial a}\right\|_{1},
$$


because both norms $\|\cdot\|_{1}$ and $\left|\|\cdot \mid\|_{\mathrm{w}_{1}}\right.$ are equivalent.

3. Unperturbed Quiescence Model. This section deals with the unperturbed Quiescence Model $(\mathrm{PQ})_{2}$ (with $\sigma=\delta=0$ ) and $(\mathrm{PQ})_{4}$, governed by the following unbounded linear operator:

$$
\mathrm{T}_{0} \varphi:=-\frac{\partial \varphi}{\partial a} \quad \text { on } \quad \mathrm{D}_{0}:=\left\{\varphi \in \mathrm{W}_{1}: \gamma_{0} \varphi=0\right\}
$$

Note that the domain $\mathrm{D}_{0}$ is well defined because of Lemma 1 .

Lemma 2. Let $\lambda>0$. Then $\left(\lambda-\mathrm{T}_{0}\right)^{-1}$ is a bounded linear operator from $\mathrm{L}_{1}$ into itself, and satisfies for all $g \in \mathrm{L}_{1}$ the inequality

$$
\begin{aligned}
& \left\|\left(\lambda-\mathrm{T}_{0}\right)^{-1} g\right\|_{1} \leqslant \frac{1}{\lambda}\|g\|_{1}, \\
& \left\|\frac{1}{l}\left(\lambda-\mathrm{T}_{0}\right)^{-1} g\right\|_{1} \leqslant\|g\|_{1} .
\end{aligned}
$$

Moreover, $\mathrm{T}_{0}$ generates a $C_{0}$-semigroup of contractions on $\mathrm{L}_{1}$.

Proof. Let $\lambda>0$ and $g \in \mathrm{L}_{1}$. Easy computations show that

$$
\left(\lambda-\mathrm{T}_{0}\right)^{-1} g(a, l)=\int_{0}^{a} e^{-\lambda\left(a-a^{\prime}\right)} g\left(a^{\prime}, l\right) \mathrm{d} a^{\prime}, \quad(a, l) \in \Omega .
$$

Let $G=\left(\lambda-\mathrm{T}_{0}\right)^{-1} g$. Firstly

$$
\|G\|_{1} \leqslant \int_{l_{1}}^{l_{2}}\left\{\int_{0}^{l}\left[e^{-\lambda a}\right]\left[\int_{0}^{a} e^{\lambda a^{\prime}}\left|g\left(a^{\prime}, l\right)\right| \mathrm{d} a^{\prime}\right] \mathrm{d} a\right\} \mathrm{d} l .
$$

Integrating by parts the term in brackets leads to

$$
\|G\|_{1} \leqslant \frac{1}{\lambda} \int_{l_{1}}^{l_{2}}\left\{-\int_{0}^{l} e^{-\lambda\left(l-a^{\prime}\right)}\left|g\left(a^{\prime}, l\right)\right| \mathrm{d} a^{\prime}+\int_{0}^{l}|g(a, l)| \mathrm{d} a\right\} \mathrm{d} l \leqslant \frac{1}{\lambda}\|g\|_{1}
$$

which proves (5) and leads to the desired boundedness. Next,

$$
\left\|\frac{1}{l} G\right\|_{1} \leqslant \int_{l_{1}}^{l_{2}}\left[\frac{1}{l} \int_{0}^{l} \mathrm{~d} a\right] \int_{0}^{l}\left|g\left(a^{\prime}, l\right)\right| \mathrm{d} a^{\prime} \mathrm{d} l=\|g\|_{1}
$$


which shows (6).

Firstly, the boundedness of $\left(\lambda-\mathrm{T}_{0}\right)^{-1}$ yields that $\left(\lambda-\mathrm{T}_{0}\right)$ is closed and so is $\mathrm{T}_{0}=\lambda\left(\lambda-\mathrm{T}_{0}\right)$. Also, $\mathrm{T}_{0}$ is densely defined because of $\mathcal{C}_{c}(\Omega) \subset \mathrm{D}_{0} \subset \mathrm{L}_{1}$, where $\mathcal{C}_{c}(\Omega)$ denotes the subspace of all continuous functions with compact support (in $\Omega$ ). Next, (5) leads, by an easy induction, to

$$
\left\|\left(\lambda-\mathrm{T}_{0}\right)^{-n} g\right\|_{1} \leqslant \frac{1}{\lambda^{n}}\|g\|_{1} \quad n=1,2,3, \ldots
$$

All required conditions of the Hille-Yosida Theorem ([1, Th. 3.5]) are now satisfied.

4. Unperturbed Proliferation Model. This section concerns the unperturbed Proliferation Model $(\mathrm{PQ})_{1}($ with $\mu=\sigma=\delta=0$ and $\eta=0$ ) and $(\mathrm{PQ})_{3}$ governed by the following unbounded linear operator:

$$
\mathrm{T}_{\alpha, \beta} \varphi:=-\frac{\partial \varphi}{\partial a} \quad \text { on } \quad \mathrm{D}_{\alpha, \beta}:=\left\{\varphi \in \mathrm{W}_{1}: \gamma_{0} \varphi=\mathrm{K}_{\alpha, \beta} \gamma_{1} \varphi\right\}
$$

where $\alpha \geqslant 0$ and $\beta \geqslant 0$ denote the average number of daughter cells viable per mitosis. Unless otherwise stated, $\alpha$ and $\beta$ are assumed to be fixed. The mitosis operator $\mathrm{K}_{\alpha, \beta}$ is defined by

$$
\mathrm{K}_{\alpha, \beta} \psi(l):=\alpha \psi(l)+\beta \int_{l_{1}}^{l_{2}} \mathrm{k}\left(l, l^{\prime}\right) \psi\left(l^{\prime}\right) \mathrm{d} l^{\prime}, \quad l \in\left(l_{1}, l_{2}\right),
$$

whose kernel $\mathrm{k}=\mathrm{k}\left(l, l^{\prime}\right)$ is assumed to be subject to the following assumption:

$$
\begin{gathered}
\left(\mathbf{A}_{\mathrm{k}}\right) \quad \kappa\left(l_{2}\right)<\infty \\
\text { where } \quad \kappa(\omega):=\operatorname{ess~sup}_{l_{1} \leqslant l^{\prime} \leqslant \omega} \int_{l_{1}}^{l_{2}}\left|\mathrm{k}\left(l, l^{\prime}\right)\right| \mathrm{d} l .
\end{gathered}
$$

Note that the domain $\mathrm{D}_{\alpha, \beta}$ is well defined because of Lemma 1 together with the following lemma:

Lemma 3. If $\left(\mathbf{A}_{\mathrm{k}}\right)$ holds, then $\mathrm{K}_{\alpha, \beta}$ is a bounded linear operator from $\mathrm{Y}_{1}$ into itself, satisfying

$$
\left\|\mathrm{K}_{\alpha, \beta}\right\|_{\mathcal{L}\left(\mathrm{Y}_{1}\right)} \leqslant \alpha+\beta \kappa\left(l_{2}\right) .
$$


Proof. For all $\psi \in \mathrm{Y}_{1}$, we have

$$
\begin{aligned}
\left\|\mathrm{K}_{\alpha, \beta} \psi\right\|_{\mathrm{Y}_{1}} & \leqslant \alpha \int_{l_{1}}^{l_{2}}|\psi(l)| \mathrm{d} l+\beta \int_{l_{1}}^{l_{2}}\left[\int_{l_{1}}^{l_{2}}\left|\mathrm{k}\left(l, l^{\prime}\right)\right| \mathrm{d} l\right]\left|\psi\left(l^{\prime}\right)\right| \mathrm{d} l^{\prime} \leqslant \\
& \leqslant \alpha \int_{l_{1}}^{l_{2}}|\psi(l)| \mathrm{d} l+\beta \kappa\left(l_{2}\right) \int_{l_{1}}^{l_{2}}\left|\psi\left(l^{\prime}\right)\right| \mathrm{d} l^{\prime},
\end{aligned}
$$

which proves (9) and leads to the desired boundedness.

As we have pointed out in the introduction, we must separate the two cases $l_{1}=0$ and $l_{1}>0$.

Lemma 4. Suppose that $l_{1}>0$. If $\left(\mathbf{A}_{\mathrm{k}}\right)$ holds, then $\left(\lambda-\mathrm{T}_{\alpha, \beta}\right)^{-1}$ $\left(\lambda>\frac{1}{l_{1}} \ln \mathrm{M}_{\alpha, \beta}\right)$ is a bounded linear operator from $\mathrm{L}_{1}$ into itself, satisfying, for all $g \in \mathrm{L}_{1}$,

$$
\left\|\left(\lambda-\mathrm{T}_{\alpha, \beta}\right)^{-n} g\right\|_{1} \leqslant \frac{\mathrm{M}_{\alpha, \beta}\|g\|_{1}}{\left(\lambda-\frac{1}{l_{1}} \ln \mathrm{M}_{\alpha, \beta}\right)^{n}}, \quad n=1,2,3, \ldots
$$

where $\mathrm{M}_{\alpha, \beta}=\max \left\{\alpha+\beta \kappa\left(l_{2}\right) ; 1\right\}$. Moreover, $\mathrm{T}_{\alpha, \beta}$ generates the $C_{0}$-semigroup $\left(\mathrm{T}_{\alpha, \beta}(t)\right)_{t \geqslant 0}$ satisfying, for all $\varphi \in \mathrm{L}_{1}$,

$$
\left\|\mathrm{T}_{\alpha, \beta}(t) \varphi\right\|_{1} \leqslant \mathrm{M}_{\alpha, \beta}\left(1+\frac{t}{l_{1}}\right)\|\varphi\|_{1} \quad t \geqslant 0 .
$$

Proof. Step I. Let $\lambda \geqslant 0$ and $\psi \in \mathrm{Y}_{1}$. Let $\mathcal{K}_{\alpha, \beta, \lambda}$ be such that

$$
\mathcal{K}_{\alpha, \beta, \lambda} \psi=\alpha e^{-\lambda \cdot} \psi+\beta \int_{l_{1}}^{l_{2}} e^{-\lambda l^{\prime}} \mathrm{k}\left(\cdot, l^{\prime}\right) \psi\left(l^{\prime}\right) \mathrm{d} l^{\prime} .
$$

As $\mathcal{K}_{\alpha, \beta, \lambda} \psi=\mathrm{K}_{\alpha, \beta}\left(e^{-\lambda} \psi\right),(9)$ implies that

$$
\left\|\mathcal{K}_{\alpha, \beta, \lambda}\right\|_{\mathcal{L}\left(\mathrm{Y}_{1}\right)} \leqslant e^{-\lambda l_{1}}\left(\alpha+\beta \kappa\left(l_{2}\right)\right)
$$

which proves that $\mathcal{K}_{\alpha, \beta, \lambda}$ is bounded from $\mathrm{Y}_{1}$ into itself and

$$
\left\|\mathcal{K}_{\alpha, \beta, \lambda}\right\|_{\mathcal{L}\left(\mathrm{Y}_{1}\right)}<1 \quad \text { for all } \quad \lambda>\frac{1}{l_{1}} \ln \mathrm{M}_{\alpha, \beta} .
$$


Step II. Let $\lambda>\frac{1}{l_{1}} \ln \mathrm{M}_{\alpha, \beta}$ and $g \in \mathrm{L}_{1}$. Let us find the solution of $\left(\lambda-\mathrm{T}_{\alpha, \beta}\right) \varphi=g$; that is,

$$
\begin{aligned}
\lambda \varphi & =-\frac{\partial \varphi}{\partial a}+g, \\
\gamma_{0} \varphi & =\mathrm{K}_{\alpha, \beta} \gamma_{1} \varphi .
\end{aligned}
$$

So, the general solution of (13) is given by

$$
\varphi(a, l)=\left(e^{-\lambda \cdot} \otimes \psi\right)(a, l)+\left(\lambda-\mathrm{T}_{0}\right)^{-1} g(a, l), \quad(a, l) \in \Omega,
$$

where $\psi \in \mathrm{Y}_{1}$. Integrating (15) and then using (5) lead to

$$
\|\varphi\|_{1} \leqslant \int_{l_{1}}^{l_{2}}\left[\int_{0}^{l} e^{-\lambda a} \mathrm{~d} a\right]|\psi(l)| \mathrm{d} l+\frac{1}{\lambda}\|g\|_{1} \leqslant \frac{1}{\lambda}\|\psi\|_{\mathrm{Y}_{1}}+\frac{1}{\lambda}\|g\|_{1}<\infty
$$

which gives, by virtue of (13),

$$
\left\|\frac{\partial \varphi}{\partial a}\right\|_{1} \leqslant \lambda\|\varphi\|_{1}+\|g\|_{1} \leqslant\|\psi\|_{\mathrm{Y}_{1}}+2\|g\|_{1}<\infty .
$$

Similarly,

$$
\left\|\frac{1}{l} \varphi\right\|_{1} \leqslant \int_{l_{1}}^{l_{2}}\left[\frac{1}{l} \int_{0}^{l} e^{-\lambda a} \mathrm{~d} a\right]|\psi(l)| \mathrm{d} l+\|g\|_{1} \leqslant\|\psi\|_{\mathrm{Y}_{1}}+\|g\|_{1}<\infty
$$

where we have used (6). Hence, $\varphi \in \mathrm{W}_{1}$.

Next, $\varphi$ satisfies (14) iff $\psi=\mathcal{K}_{\alpha, \beta, \lambda} \psi+\mathrm{K}_{\alpha, \beta} \gamma_{1}\left(\lambda-\mathrm{T}_{0}\right)^{-1} g$ which leads, by $(12)$, to $\psi=\left(\mathbb{I}-\mathcal{K}_{\alpha, \beta, \lambda}\right)^{-1} \mathrm{~K}_{\alpha, \beta} \gamma_{1}\left(\lambda-\mathrm{T}_{0}\right)^{-1} g$. Putting this into (15), we finally get

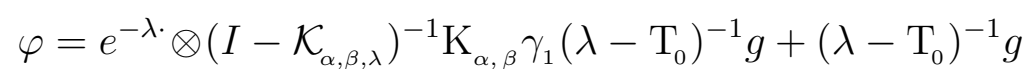

which is the unique solution of $\left(\lambda-\mathrm{T}_{\alpha, \beta}\right) \varphi=g$. Hence,

$$
\left(\lambda-\mathrm{T}_{\alpha, \beta}\right)^{-1} g=\varphi \quad \text { for all } \quad \lambda>\frac{1}{l_{1}} \ln \mathrm{M}_{\alpha, \beta} .
$$

Step III. Firstly, let us consider the following norm on $\mathrm{L}_{1}$ :

$$
\||g|\|_{1}=\int_{\Omega}|g(a, l)| \mathrm{M}_{\alpha, \beta}^{\frac{a}{l}} \mathrm{~d} a \mathrm{~d} l
$$


which is equivalent to the norm $\|\cdot\|_{1}$ because of

$$
\|g\|_{1} \leqslant\left\|\left|\left\|g||_{1} \leqslant \mathrm{M}_{\alpha, \beta}\right\| g \|_{1} \quad \text { for all } g \in \mathrm{L}_{1}\right.\right. \text {. }
$$

Next, let $\lambda>\frac{1}{l_{1}} \ln \mathrm{M}_{\alpha, \beta}$ and $g \in \mathrm{L}_{1}$. Multiplying both sides of (13) by $(\operatorname{sgn} \varphi)(a, l) \mathrm{M}_{\alpha, \beta}^{\frac{a}{l}}$ and then integrating over $\Omega$ lead to

$$
\lambda\left|\|\varphi\|_{1} \leqslant \int_{\Omega} \mathrm{M}_{\alpha, \beta}^{\frac{a}{l}} \frac{\partial|\varphi|}{\partial a}(a, l) \mathrm{d} a \mathrm{~d} l+\right|\left\|g\left|\left\|_{1}:=I+\right\|\right||g|\right\|_{1} .
$$

Integrating by parts, the term $I$ is transformed to

$$
\begin{aligned}
I & =-\int_{\Omega} \frac{\partial\left(\mathrm{M}_{\alpha, \beta}^{\frac{a}{l}}|\varphi|\right)}{\partial a}(a, l) \mathrm{d} a \mathrm{~d} l+\ln \mathrm{M}_{\alpha, \beta} \int_{\Omega} \frac{1}{l} \mathrm{M}_{\alpha, \beta}^{\frac{a}{l}}|\varphi(a, l)| \mathrm{d} a \mathrm{~d} l \leqslant \\
& \leqslant-\mathrm{M}_{\alpha, \beta} \int_{l_{1}}^{l_{2}}\left|\gamma_{1} \varphi(l)\right| \mathrm{d} l+\int_{l_{1}}^{l_{2}}\left|\gamma_{0} \varphi(l)\right| \mathrm{d} l+\left.\frac{1}{l_{1}} \ln \mathrm{M}_{\alpha, \beta}|||\varphi|\right|_{1},
\end{aligned}
$$

which leads, by virtue of (14) and then (9), to

$$
I \leqslant\left(\left\|\mathrm{~K}_{\alpha, \beta}\right\|-\mathrm{M}_{\alpha, \beta}\right)\left\|\gamma_{1} \varphi\right\|_{\mathrm{Y}_{1}}+\frac{1}{l_{1}} \ln \mathrm{M}_{\alpha, \beta}\left|\left\|\varphi \left|\left\|\left.\right|_{1} \leqslant \frac{1}{l_{1}} \ln \mathrm{M}_{\alpha, \beta} \mid\right\| \varphi\|\|_{1} .\right.\right.\right.
$$

Combining this together with (19) and then (17) yields

$$
\|\|\left(\lambda-\mathrm{T}_{\alpha, \beta}\right)^{-1} g \mid \|_{1} \leqslant \frac{\|\| g \mid \|_{1}}{\left(\lambda-\frac{1}{l_{1}} \ln \mathrm{M}_{\alpha, \beta}\right)}
$$

which leads, by an easy induction on the integer $n \geqslant 1$, to

$$
\|\|\left(\lambda-\mathrm{T}_{\alpha, \beta}\right)^{-n} g \|_{1} \leqslant \frac{\|\| g\|\|_{1}}{\left(\lambda-\frac{1}{l_{1}} \ln \mathrm{M}_{\alpha, \beta}\right)^{n}} \quad n=1,2,3, \ldots
$$

and by (18),

$$
\left\|\left(\lambda-\mathrm{T}_{\alpha, \beta}\right)^{-n} g\right\|_{1} \leqslant \frac{\mathrm{M}_{\alpha, \beta}}{\left(\lambda-\frac{1}{l_{1}} \ln \mathrm{M}_{\alpha, \beta}\right)^{n}}\|g\|_{1} \quad n=1,2,3, \ldots
$$


Hence, (10) follows and proves the boundedness of $\left(\lambda-\mathrm{T}_{\alpha, \beta}\right)^{-1}$ for $n=1$.

Step IV. The boundedness of $\left(\lambda-\mathrm{T}_{\alpha, \beta}\right)^{-1}$ yields that $\left(\lambda-\mathrm{T}_{\alpha, \beta}\right)$ is closed and so is $\mathrm{T}_{\alpha, \beta}=\lambda-\left(\lambda-\mathrm{T}_{\alpha, \beta}\right) . \quad \mathrm{T}_{\alpha, \beta}$ is densely defined because of $\mathcal{C}_{c}(\Omega) \subset \mathrm{D}_{\alpha, \beta} \subset \mathrm{L}_{1}$. Now, all required conditions of the Hille-Yosida Theorem ( $[1$, Th. 3.5]) are satisfied.

The previous study of the case $l_{1}>0$ can not be extended to the case $l_{1}=0$ because, for instance, (12) is no longer valid when $l_{1}=0$. Accordingly, the study of the case $l_{1}=0$ needs an additional assumption. Let us consider
$\left(\mathbf{A}_{\mathrm{k}}^{\prime}\right)$
$\exists \omega_{0} \in\left(0, l_{2}\right) \quad: \quad \alpha+\beta \kappa\left(\omega_{0}\right)<1$

where $\kappa$ is defined by (8). As $\alpha+\beta \kappa(\omega) \leqslant \alpha+\beta \kappa\left(l_{2}\right)$ for all $\omega \in\left(0, l_{2}\right)$, $\left(\mathbf{A}_{\mathrm{k}}^{\prime}\right)$ holds whenever $\left(\mathbf{A}_{\mathrm{k}}\right)$ holds and $\alpha+\beta \kappa\left(l_{2}\right)<1$ (the contractiveness case). However, in the general case we have

Lemma 5. Suppose that $l_{1}=0$. Also suppose that $\left(\mathbf{A}_{\mathrm{k}}\right)$ holds. If $\left(\mathbf{A}_{\mathrm{k}}^{\prime}\right)$ holds, then $\left(\lambda-\mathrm{T}_{\alpha, \beta}\right)^{-1}\left(\lambda>\frac{1}{\omega_{0}} \ln \mathrm{M}_{\alpha, \beta}\right)$ is a bounded linear operator from $\mathrm{L}_{1}$ into itself and satisfies

$$
\left\|\left(\lambda-\mathrm{T}_{\alpha, \beta}\right)^{-n} g\right\|_{1} \leqslant \frac{\mathrm{M}_{\alpha, \beta}\|g\|_{1}}{\left(\lambda-\frac{1}{\omega_{0}} \ln \mathrm{M}_{\alpha, \beta}\right)^{n}} \quad n=1,2,3, \ldots
$$

for all $g \in \mathrm{L}_{1}$, where $\mathrm{M}_{\alpha, \beta}$ is defined in Lemma 4 and $\omega_{0}$ is given in $\left(\mathbf{A}_{\mathrm{k}}^{\prime}\right)$. Moreover, $\mathrm{T}_{\alpha, \beta}$ generates a $C_{0}$-semigroup $\left(\mathrm{T}_{\alpha, \beta}(t)\right)_{t \geqslant 0}$ on $\mathrm{L}_{1}$ satisfying for all $\varphi \in \mathrm{L}_{1}$

$$
\left\|\mathbb{T}_{\alpha, \beta}(t) \varphi\right\|_{1} \leqslant \mathrm{M}_{\alpha, \beta}\left(1+\frac{t}{\omega_{0}}\right)\|\varphi\|_{1} \quad t \geqslant 0
$$

Proof. Step I. Let $\lambda \geqslant 0$ and $\psi \in \mathrm{Y}_{1}$. Since (11), we can write

$$
\begin{aligned}
& \left\|\mathcal{K}_{\alpha, \beta, \lambda} \psi\right\|_{\mathrm{Y}_{1}} \leqslant \alpha \int_{0}^{l_{2}} e^{-\lambda l}|\psi(l)| \mathrm{d} l+\beta \int_{0}^{l_{2}} \int_{0}^{l_{2}} e^{-\lambda l^{\prime}}\left|\mathrm{k}\left(l, l^{\prime}\right)\right|\left|\psi\left(l^{\prime}\right)\right| \mathrm{d} l^{\prime} \mathrm{d} l \leqslant \\
& \leqslant \alpha \int_{0}^{\omega_{0}}|\psi(l)| \mathrm{d} l+\alpha e^{-\lambda \omega_{0}} \int_{\omega_{0}}^{l_{2}}|\psi(l)| \mathrm{d} l+\beta \int_{0}^{\omega_{0}}\left[\int_{0}^{l_{2}}\left|\mathrm{k}\left(l, l^{\prime}\right)\right| \mathrm{d} l\right]\left|\psi\left(l^{\prime}\right)\right| \mathrm{d} l^{\prime}+
\end{aligned}
$$




$$
\begin{aligned}
& +\beta e^{-\lambda \omega_{0}} \int_{\omega_{0}}^{l_{2}}\left[\int_{0}^{l_{2}}\left|\mathrm{k}\left(l, l^{\prime}\right)\right| \mathrm{d} l\right]\left|\psi\left(l^{\prime}\right)\right| \mathrm{d} l^{\prime} \leqslant \\
& \leqslant \max \left\{\left(\alpha+\beta \kappa\left(\omega_{0}\right)\right), e^{-\lambda \omega_{0}}\left(\alpha+\beta \kappa\left(l_{2}\right)\right)\right\}\|\psi\|_{\mathrm{Y}_{1}},
\end{aligned}
$$

which proves that $\mathcal{K}_{\alpha, \beta, \lambda}$ is bounded from $\mathrm{Y}_{1}$ into itself and

$$
\left\|\mathcal{K}_{\alpha, \beta, \lambda}\right\|_{\mathcal{L}\left(\mathrm{Y}_{1}\right)} \leqslant \max \left\{\left(\alpha+\beta \kappa\left(\omega_{0}\right)\right), e^{-\lambda \omega_{0}}\left(\alpha+\beta \kappa\left(l_{2}\right)\right)\right\} .
$$

Now $\left(\mathbf{A}_{\mathrm{k}}^{\prime}\right)$ yields that

$$
\left\|\mathcal{K}_{\alpha, \beta, \lambda}\right\|_{\mathcal{L}\left(\mathrm{Y}_{1}\right)}<1 \quad \text { for all } \quad \lambda>\frac{1}{\omega_{0}} \ln \mathrm{M}_{\alpha, \beta} .
$$

Step II. Let $\lambda>\frac{1}{\omega_{0}} \ln \mathrm{M}_{\alpha, \beta}$ and $g \in \mathrm{L}_{1}$. Firstly, following step II of the proof of Lemma 4 and using (21) instead of (12), we can similarly prove that (16) is the unique solution of the equation $\left(\lambda-\mathrm{T}_{\alpha, \beta}\right) \varphi=g$ and, therefore,

$$
\left(\lambda-\mathrm{T}_{\alpha, \beta}\right)^{-1} g=\varphi \quad \text { for all } \quad \lambda>\frac{1}{\omega_{0}} \ln \mathrm{M}_{\alpha, \beta} .
$$

Next, let us consider the following norm on $\mathrm{L}_{1}$ :

$$
\||g|\|_{1}=\int_{\Omega}|g(a, l)| \mathrm{M}_{\alpha, \beta} \min \left\{\frac{a}{\omega_{0}}, 1\right\} \mathrm{d} a \mathrm{~d} l
$$

which is equivalent to the norm $\|\cdot\|$ because of

$$
\|g\|_{1} \leqslant\|\| g\|\|_{1} \leqslant \mathrm{M}_{\alpha, \beta}\|g\|_{1} \quad \text { for all } \quad g \in \mathrm{L}_{1} .
$$

Following the step III of the proof of Lemma 4, we get

$$
\left\|\left(\lambda-\mathrm{T}_{\alpha, \beta}\right)^{-n} g\right\| \|_{1} \leqslant \frac{\|\| g\|\|_{1}}{\left(\lambda-\frac{1}{\omega_{0}} \ln \mathrm{M}_{\alpha, \beta}\right)^{n}} \quad n=1,2,3, \ldots
$$

and

$$
\left\|\left(\lambda-\mathrm{T}_{\alpha, \beta}\right)^{-n} g\right\|_{1} \leqslant \frac{\mathrm{M}_{\alpha, \beta}\|g\|_{1}}{\left(\lambda-\frac{1}{\omega_{0}} \ln \mathrm{M}_{\alpha, \beta}\right)^{n}} \quad n=1,2,3, \ldots
$$


due to (22). Hence, (20) follows and proves the boundedness of $\left(\lambda-\mathrm{T}_{\alpha, \beta}\right)^{-1}$ for $n=1$.

Step III. This step is similar to the step IV of the proof of Lemma 4.

5. Unperturbed Proliferation-Quiescence Model. This section deals with the unperturbed Proliferation-Quiescence Model (PQ) $)_{1}$ (with $\mu=\sigma=\delta=0$ and $\eta=0),(\mathrm{PQ})_{2}($ with $\sigma=\delta=0),(\mathrm{PQ})_{3}$ and $(\mathrm{PQ})_{4}$ governed by the following unbounded linear operator:

$$
\mathrm{U}_{\alpha, \beta}:=\left(\begin{array}{cc}
\mathrm{T}_{\alpha, \beta} & 0 \\
0 & \mathrm{~T}_{0}
\end{array}\right) \text { on the domain } \mathrm{D}_{\alpha, \beta} \times \mathrm{D}_{\alpha, \beta},
$$

where $\mathrm{T}_{\alpha, \beta}$ and $\mathrm{T}_{0}$ are already studied in the previous sections. Let $\mathrm{X}_{1}$ be the following Banach space

$$
\mathrm{X}_{1}:=\mathrm{L}_{1} \times \mathrm{L}_{1} \quad \text { whose norm is }\left\|\left(\begin{array}{l}
\psi \\
\phi
\end{array}\right)\right\|_{\mathrm{X}_{1}}:=\|\varphi\|_{1}+\|\phi\|_{1} .
$$

The first aim of this section deals with the case $l_{1}>0$.

Theorem 1. Suppose that $l_{1}>0$. If $\left(\mathbf{A}_{\mathrm{k}}\right)$ holds, $\mathrm{U}_{\alpha, \beta}$ generates a $C_{0}-$ semigroup $\left(\mathbb{U}_{\alpha, \beta}(t)\right)_{t \geqslant 0}$ on $\mathrm{X}_{1}$; it satisfies

$$
\left\|\mathbb{U}_{\alpha, \beta}(t)\right\|_{\mathcal{L}\left(\mathrm{X}_{1}\right)} \leqslant \mathrm{M}_{\alpha, \beta}\left(1+\frac{t}{l_{1}}\right) \quad t \geqslant 0,
$$

where $\mathrm{M}_{\alpha, \beta}$ is defined in Lemma 4.

Proof. Let $\lambda>\frac{1}{l_{1}} \ln \mathrm{M}_{\alpha, \beta}$. Lemmas 4 and 2 yield

$$
\left(\lambda-\mathrm{U}_{\alpha, \beta}\right)^{-1}=\left(\begin{array}{cc}
\left(\lambda-\mathrm{T}_{\alpha, \beta}\right)^{-1} & 0 \\
0 & \left(\lambda-\mathrm{T}_{0}\right)^{-1}
\end{array}\right)
$$

and by induction

$$
\left(\lambda-\mathrm{U}_{\alpha, \beta}\right)^{-n}=\left(\begin{array}{cc}
\left(\lambda-\mathrm{T}_{\alpha, \beta}\right)^{-n} & 0 \\
0 & \left(\lambda-\mathrm{T}_{0}\right)^{-n}
\end{array}\right) \quad n=1,2, \ldots
$$

Let $n \geqslant 1$ be an integer and let $\left(\begin{array}{l}\psi \\ \phi\end{array}\right) \in \mathrm{X}_{1}$. Since (10) and (5), we get

$$
\left\|\left(\lambda-\mathrm{U}_{\alpha, \beta}\right)^{-n}\left(\begin{array}{l}
\psi \\
\phi
\end{array}\right)\right\|_{\mathrm{x}_{1}}=\left\|\left(\lambda-\mathrm{T}_{\alpha, \beta}\right)^{-n} \phi\right\|_{1}+\left\|\left(\lambda-\mathrm{T}_{0}\right)^{-n} \phi\right\|_{1} \leqslant
$$




$$
\leqslant \frac{\mathrm{M}_{\alpha, \beta}}{\left(\lambda-\frac{1}{l_{1}} \ln \mathrm{M}_{\alpha, \beta}\right)^{n}}\left\|\left(\begin{array}{c}
\psi \\
\phi
\end{array}\right)\right\|_{\mathrm{x}_{1}}
$$

and, therefore,

$$
\left\|\left(\lambda-\mathrm{U}_{\alpha, \beta}\right)^{-n}\right\|_{\mathcal{L}\left(\mathrm{x}_{1}\right)} \leqslant \frac{\mathrm{M}_{\alpha, \beta}}{\left(\lambda-\frac{1}{l_{1}} \ln \mathrm{M}_{\alpha, \beta}\right)^{n}} \quad n=1,2,3, \ldots
$$

As $\left(\lambda-\mathrm{U}_{\alpha, \beta}\right)^{-1}$ is bounded, $\left(\lambda-\mathrm{U}_{\alpha, \beta}\right)$ is closed, and so is $\mathrm{U}_{\alpha, \beta}=\lambda-\left(\lambda-\mathrm{U}_{\alpha, \beta}\right)$. Furthermore, $\overline{\mathrm{D}_{\alpha, \beta} \times \mathrm{D}_{0}}=\overline{\mathrm{D}_{\alpha, \beta}} \times \overline{\mathrm{D}_{0}}=\mathrm{L}_{1} \times \mathrm{L}_{1}=\mathrm{X}_{1}$. Now all the required conditions of the Hille-Yosida Theorem ( [1, Th. 3.5]) are satisfied.

The second aim of this section deals with the case $l_{1}=0$.

Theorem 2. Suppose that $l_{1}=0$. Suppose, furthermore, that $\left(\mathbf{A}_{\mathrm{k}}\right)$ holds. If $\left(\mathbf{A}_{\mathrm{k}}^{\prime}\right)$ holds, $\mathrm{U}_{\alpha, \beta}$ generates a $C_{0}-\operatorname{semigroup}\left(\mathbb{U}_{\alpha, \beta}(t)\right)_{t \geqslant 0}$ on $\mathrm{X}_{1}$; it satisfies

$$
\left\|\mathbb{U}_{\alpha, \beta}(t)\right\|_{\mathcal{L}\left(\mathrm{X}_{1}\right)} \leqslant \mathrm{M}_{\alpha, \beta}\left(1+\frac{t}{\omega_{0}}\right) \quad t \geqslant 0,
$$

where $\mathrm{M}_{\alpha, \beta}$ is defined in Lemma 4 and $\omega_{0}$ is given in $\left(\mathbf{A}_{\mathrm{k}}^{\prime}\right)$.

Proof. The proof is similar to the proof of Theorem 1. Due to (20) and (5), we easily get

$$
\left\|\left(\lambda-\mathrm{U}_{\alpha, \beta}\right)^{-n}\right\|_{\mathcal{L}\left(\mathrm{x}_{1}\right)} \leqslant \frac{\mathrm{M}_{\alpha, \beta}}{\left(\lambda-\frac{1}{\omega_{0}} \ln \mathrm{M}_{\alpha, \beta}\right)^{n}} \quad n=1,2,3, \ldots
$$

Now the Hille-Yosida Theorem ( [1, Th. 3.5]) ends the proof.

6. Full Proliferation-Quiescence Model. The aim of this section is the well posedness of the full Proliferation-Quiescence Model (PQ) ${ }_{1}^{-}$ $(\mathrm{PQ})_{4}$ governed by the following unbounded linear operator:

$$
\mathrm{V}_{\alpha, \beta}:=\mathrm{U}_{\alpha, \beta}+\mathrm{B} \quad \text { on the domain } \quad \mathrm{D}_{\alpha, \beta} \times \mathrm{D}_{0}
$$

with

$$
\mathrm{B}:=\left(\begin{array}{cc}
-\sigma \mathbb{I}-\mu \mathbb{I}+\mathrm{R} & \delta \mathbb{I} \\
\sigma \mathbb{I} & -\delta \mathbb{I}
\end{array}\right)
$$


where I denotes the identity operator in $\mathrm{L}_{1}$ and

$$
\mathrm{R} \varphi(a, l):=\int_{l_{1}}^{l_{2}} \eta\left(a, l, l^{\prime}\right) \varphi\left(a, l^{\prime}\right) \mathrm{d} l^{\prime}, \quad(a, l) \in \Omega .
$$

Suppose that the rates $\mu, \sigma$ and $\delta$, and the kernel $r$ are subject to the following assumptions
$\left(\mathbf{A}_{\sigma}\right):$
$\operatorname{ess} \sup |\sigma(a, l)|<\infty$
$(a, l) \in \Omega$
$\left(\mathbf{A}_{\delta}\right):$
$\operatorname{ess} \sup |\delta(a, l)|<\infty$
$(a, l) \in \Omega$
$\left(\mathbf{A}_{\mu}\right):$
$\operatorname{ess} \sup |\mu(a, l)|<\infty$,
$(a, l) \in \Omega$
$\left(\mathbf{A}_{\eta}\right):$
$\underset{\left(a, l^{\prime}\right) \in \Omega}{\operatorname{ess} \sup _{l_{1}}} \int_{l_{2}}^{l_{2}}\left|\eta\left(a, l, l^{\prime}\right)\right| \mathrm{d} l<\infty$.

Lemma 6. Suppose that the assumptions $\left(\mathbf{A}_{\sigma}\right),\left(\mathbf{A}_{\delta}\right),\left(\mathbf{A}_{\mu}\right)$ and $\left(\mathbf{A}_{\eta}\right)$ hold. Then $\mathrm{B}$ is a bounded linear operator from $\mathrm{X}_{1}$ into itself.

Proof. Firstly, the assumption $\left(\mathbf{A}_{\mu}\right)$ yields that the multiplicative operator $\mu \mathbb{I}$ is bounded from $\mathrm{L}_{1}$ into itself. Similarly, the multiplicative operators $\sigma \mathbb{I}$ and $\delta \mathbb{I}$ are also bounded from $\mathrm{L}_{1}$ into itself. It remains to prove that $\mathrm{R}$ is a bounded linear operator from $\mathrm{L}_{1}$ into itself. So, for all $\varphi \in \mathrm{L}_{1}$, we have

$$
\begin{aligned}
\|\mathrm{R} \varphi\|_{1} & \leqslant \iint_{\Omega}\left|\int_{l_{1}}^{l_{2}}\right| \eta\left(a, l, l^{\prime}\right)|| \varphi\left(a, l^{\prime}\right)\left|\mathrm{d} l^{\prime}\right| \mathrm{d} a \mathrm{~d} l \leqslant \\
& \leqslant\left[\underset{\left(a, l^{\prime}\right) \in \Omega}{\operatorname{ess} \sup _{l_{1}}}\left|\eta\left(a, l, l^{\prime}\right)\right| \mathrm{d} l\right] \int_{\Omega}\left|\varphi\left(a^{\prime}, l^{\prime}\right)\right| \mathrm{d} a^{\prime} \mathrm{d} l^{\prime},
\end{aligned}
$$

which proves the desired boundedness because of $\left(\mathbf{A}_{\eta}\right)$.

Now, due to Lemma 6, we can say that the unbounded linear operator $\mathrm{V}_{\alpha, \beta}$ is well defined and for we have

Theorem 3. Suppose that $l_{1}>0$. Suppose, furthermore, that $\left(\mathbf{A}_{\mathrm{k}}\right)$, $\left(\mathbf{A}_{\sigma}\right),\left(\mathbf{A}_{\delta}\right),\left(\mathbf{A}_{\mu}\right)$ and $\left(\mathbf{A}_{\eta}\right)$ hold. Then $\mathrm{V}_{\alpha, \beta}$ generates a $C_{0}$-semigroup on $\mathrm{X}_{1}$. 
Proof. It suffices to remark that $\mathrm{V}_{\alpha, \beta}=\mathrm{U}_{\alpha, \beta}+\mathrm{B}$ is a linear perturbation of the generator $\mathrm{U}_{\alpha, \beta}$ (Theorem 1 ) by the bounded operator B (Lemma 6). Now [1, Th. 4.9] ends the proof.

However, if $l_{1}=0$, we have

Theorem 4. Suppose that $l_{1}=0$. Suppose, furthermore, that $\left(\mathbf{A}_{\mathrm{k}}\right),\left(\mathbf{A}_{\mathrm{k}}^{\prime}\right)$, $\left(\mathbf{A}_{\sigma}\right),\left(\mathbf{A}_{\delta}\right),\left(\mathbf{A}_{\mu}\right)$ and $\left(\mathbf{A}_{\eta}\right)$ hold. Then $\mathrm{V}_{\alpha, \beta}$ generates a $C_{0}$-semigroup on $\mathrm{X}_{1}$.

Proof. The proof is similar to the proof of Theorem 3 (with Theorem 2 instead of Theorem 1).

Remark. It is easy to prove that the generated semigroups in Theorem 3 and Theorem 4 are positive provided that $\mathrm{k}, \mu, \sigma, \delta$ and $\eta$ are positive.

Remark. Our choice of $\mathrm{X}_{1}=\mathrm{L}_{1} \times \mathrm{L}_{1}$ was natural because

$$
\|f(t, \cdot, \cdot)\|_{1}=\int_{\Omega}|f(t, a, l)| \mathrm{d} a \mathrm{~d} l
$$

denotes the number of cells at time $t>0$. Nevertheless, we can extended this work to the phase space $\mathrm{X}_{p}:=L^{p}(\Omega) \times L^{p}(\Omega)(p>1)$. In this case, it suffices to replace (8) and (24) by

$$
\begin{gathered}
\kappa(\omega):=\left[\underset{l_{1} \leqslant l^{\prime} \leqslant \omega}{\operatorname{ess} u p} \int_{l_{1}}^{l_{2}}\left|\mathrm{k}\left(l, l^{\prime}\right)\right| \mathrm{d} l\right]^{\frac{1}{p}}\left[\underset{l_{1} \leqslant l \leqslant l_{2}}{\operatorname{ess} \sup _{l_{1}}}\left|\mathrm{k}\left(l, l^{\prime}\right)\right| \mathrm{d} l^{\prime}\right]^{\left(1-\frac{1}{p}\right)} \\
{\left[\underset{\left(a, l^{\prime}\right) \in \Omega}{\operatorname{ess} \int_{l_{1}}}\left|\eta\left(a, l, l^{\prime}\right)\right| \mathrm{d} l\right]^{\frac{1}{p}}\left[\underset{(a, l) \in \Omega}{l_{2}} \int_{l_{1}}^{\operatorname{esssup}_{2}}\left|\eta\left(a, l, l^{\prime}\right)\right| \mathrm{d} l^{\prime}\right]^{\left(1-\frac{1}{p}\right)}}
\end{gathered}
$$

Acknowledgment. This work is supported by LMCM-RSA.

\section{References}

[1] Banasiak J., Arlotti L. Perturbations of Positive Semigroups with Applications. Springer-Verlag London, 2006.

DOI: https://doi.org/10.1007/1-84628-153-9

[2] Boulanouar M. On a Mathematical model of Age-Cycle Length Structured Cell Population With Non-Compact Boundary Conditions. Math. Meth. Appl. Sci. 2016, vol. 39, no. 7, pp. $1855-1876$.

DOI: https://doi.org/10.1002/mma.3606 
[3] Boulanouar M. A Mathematical Analysis of a Model of Structured Population (I). Diff. Int. Equ. 2012, vol. 25, no. 9/10, pp. 821-852.

[4] Boulanouar M.. A Mathematical study in the theory of dynamic population. Journal. Math. Anal. Appl. 2001 no. 255, pp. 230-259.

DOI: https://doi.org/10.1006/jmaa.2000.7237

[5] Rotenberg M. Transport theory for growing cell populations. J. Theor. Biol. 1983, no. 103, pp. $191-199$.

DOI: https://doi.org/10.1016/0022-5193(83)90024-3

[6] Webb G. F. Dynamics of Structured Populations with Inherited Properties. Comput. Math. Applic. 1987, vol.13, no. 9-11, pp. 749-757.

DOI: https://doi.org/10.1016/0898-1221(87)90160-X

Received March 17, 2021.

In revised form, May 24, 2021.

Accepted May 29, 2021.

Published online June 13, 2021.

\section{LMCM-RSA}

22 Rue des Canadiens

Poitiers, 86000, France

boulanouar@gmail.com 